\title{
TOWARDS ENHANCING SOLUTION SPACE DiVERSITY IN MULTI-OBJECTIVE OPTIMIZATION: A HYPERVOLUME-BASED APPROACH
}

\author{
Kamyab Tahernezhadiani, Ali Hamzeh and Sattar Hashemi \\ Department of Computer Science Engineering and Information Technology, Shiraz \\ University, Shiraz, Iran \\ tahernezhadiani@cse.shirazu.ac.ir \\ ali@cse.shirazu.ac.ir \\ s.hashemieshirazu.ac.ir
}

\begin{abstract}
Diversity is an important notion in multi-objective evolutionary algorithms (MOEAs) and a lot of researchers have investigated this issue by means of appropriate methods. However most of evolutionary multi-objective algorithms have attempted to take control on diversity in the objective space only and maximized diversity of solutions (population) on Pareto- front. Nowadays due to importance of Multiobjective optimization in industry and engineering, most of the designers want to find a diverse set of Pareto-optimal solutions which cover as much as space in its feasible regain of the solution space. This paper addresses this issue and attempt to introduce a method for preserving diversity of non-dominated solution (i.e. Pareto-set) in the solution space. This paper introduces the novel diversity measure as a first time, and then this new diversity measure is integrated efficiently into the hypervolume based Multiobjective method. At end of this paper we compare the proposed method with other state-of-the-art algorithms on well-established test problems. Experimental results show that the proposed method outperforms its competitive MOEAs respect to the quality of solution space criteria and Pareto-set approximation.
\end{abstract}

\section{KEYWORDS}

Multi-objective optimization, Evolutionary algorithm, Diversity, Pareto-set and Hypervolume.

\section{INTRODUCTION}

Recently, Multi-objective optimizations (MOPs) become more popular and most of real world optimization problems are inherently a Multi-objective task [1]. In fact, Multi-objective optimization, dealing with problems that have two or more objectives which minimizing one of them led to maximizing another one. Therefore, for this problem, no single solution can fulfill all objectives simultaneously. Thus the main target of such problem is to find set of compromise solutions that neither can dominate others [2]. Although many primary approaches have been introduced to solve MOPs, like deterministic methods or arithmetic approaches, in the last few years, evolutionary algorithms (EAs) have been proved to be very popular tools to solve MOPs, since they have been able to find multiple trade-off solutions in one single run [1]. 
International Journal of Artificial Intelligence \& Applications (IJAIA), Vol.3, No.1, January 2012

In the literature, all non-dominated solutions in the objective space are called Pareto-front and all non-dominated solutions in the solution space are called Pareto-set [1]. Aims of any elocutionary based multi-objective optimizations are converging to the true Pareto-front and providing diversity of non-dominated solutions in objective and/or solution space. Most MOEAs have concentrated on diversity in the objective space only and less have attempted to enhance diversity in terms of the solution space. Therefore outcomes of existing MOEAs have enough diverse Pareto-front to satisfy designer, meanwhile population diversity of their Pareto-set is not desirable. For example, in NSGA-II as one of the state of the art MOEAs proposed by Deb et al. [3], solutions in the highest rank in sorting algorithm, only one of them survives, if it has a more distance from its neighbors in the objective space, SMS-MOEA [4] for other instance, aiming at maximizing the hypervolume indicator of obtained non-dominated solutions in the objective space. Many other methods are presented in [1] dealing with MOPs purposefully. All of which except NSGA [5], optimize indicators which compatible with Pareto-front only. In NSGA, Researchers used a niching approach for maintaining diversity of solutions in the solution space then adopted the non-dominated sorting algorithm to guide the search to the optimal solutions.

In single-objective optimization usually term of diversity referred to solution (i.e. genotype) space diversity. In such problems diversity takes an important role to obtain better results and prevents to premature convergence [6], however in multi-objective problems this issue already exists and enhancing diversity of solution space help to seek from local outcomes. Few years ago, some studies have picked up on diversity of Pareto-set as an objective in Multi-objective based methods [7-10]. But those studies are very few and because of the importance of this issue, it is a necessity to develop and introduce new approaches.

In this paper, we provide and introduce a new mechanism based on Indicator-based MOEA (i.e. SMS-EMOA [4]). Recently hypervolume-based evolutionary multi-objective algorithms have tackled by most of researchers and become suitable approaches due to their outstanding potential capabilities for fulfilling user preferences that are compliant with principle of Pareto optimality [11-12]. In those frameworks, solutions are survived and being as a parent based on their contributions respect to hypervolume [13]. The main motivation behind to this paper is acquiring set of non-dominated solutions as diverse as in the solution space. For do that, in environmental selection phase of evolutionary framework, solutions have been survived if be able to optimize both diversity and Pareto optimality indicators. In this study because of attainment to the main aim of this paper (i.e. enhancing population diversity in the Pareto- optimal set), we have adjusted parameter values and also modified some parts of algorithms proposed in [4]. In fact, we have weighted all marginal parts of generated hypervolume in the objective space by appropriate diversity indicator values of non-dominated solutions in the solution space. Our proposed method so called, weighted marginal hypervolume-multi-objective evolutionary algorithm: (WMH-MOEA) capable to obtain a set of non-dominated solutions that has a good hypervolume measure as a criterion in the objective space having a good diversity indicator as a performance measure in the solution space. Actually, our innovation is embedding the novel diversity indicator in the solution space into hypervolume criterion in the objective space.

The rest of this paper is organized as follows: in Section 2, we give a background to Multi objective evolutionary algorithms. Some related works are briefly described in section 3 . Proposed method is described in Section 4, then in Section 5 the experimental results are shown, Finally Section 6 includes conclusions and future studies have been suggested. 


\section{BACKGROUND}

In this section we describe some fundamental concepts of evolutionary multi-objective algorithm in more detail.

\subsection{Multi-objective Optimization}

Without loss of generality; an m-objective minimization problem is described as follows:

Minimize $F(x)=\left(f_{1}(x), f_{2}(x) \ldots f_{m}(x)\right)$, S.T. $x \in X$

where $\mathrm{F}(\mathrm{x})$ is the $\mathrm{m}$-dimensional objective vector, $\mathrm{f}_{\mathrm{i}}(\mathrm{x})$ is the $\mathrm{i}$-th objective to be minimized. $\mathrm{x}$ is the decision vectors belong to feasible region $\mathrm{X}$ of solution space.

\subsection{Pareto Optimality}

Let $A$ and $B$ be two feasible solutions of the m-objective minimization problem in (Equation 1). If the following conditions hold, A can be viewed as being better than B and say A dominates B or $\mathrm{B}$ is dominated byA.

$$
A \preccurlyeq B \leftrightarrow \quad \forall i \in\{1, \ldots m\}: f_{i}(A) \leq f_{i}(B) \text { and } \exists j f_{j}(A)<f_{j}(B)
$$

Figure 1 shows a graphical illustration of (Equation 2). When A is not dominated by any other feasible solutions, we say that, $\mathrm{A}$ is a non-dominated solution. Set of all non-dominated solutions in decision space, called Pareto (-optimal) set and set of all non-dominated solution in objective space, called Pareto (-optimal) front, mathematically described as follows:

$$
\begin{aligned}
& P S^{*}=\{x \in X \mid \nexists y \in X: y \preccurlyeq x\} \\
& P F^{*}=\{F(x) \mid x \in P S\}
\end{aligned}
$$

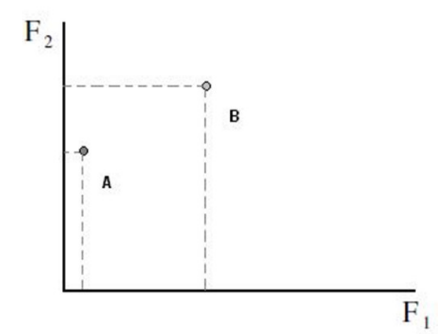

Figure 1. Graphical illustration of dominance relation

\subsection{Hypervolume}

The hypervolume or S-metric originally proposed by Zitzler et al. [11] for comparing final results of different MOEAs. Hypervolume indicator is only Pareto compliant indicator and calculates the area of fraction of the objective space that is dominated by induced Pareto-front and none dominated by specific vector (i.e. reference point) in terms of the objective space. In mathematical description, the hypervolume indicator can be shown as follows: 
International Journal of Artificial Intelligence \& Applications (IJAIA), Vol.3, No.1, January 2012

Hypervolume $\left(\right.$ Set $\left.S, s_{\text {ref }}\right)=\operatorname{VOL}\left(\mathrm{U}_{S}\left\{s^{\prime} \mid s \leqslant s^{\prime} \leqslant s_{\text {ref }}\right\}\right)$

where VOL (.) denotes the Lebesgue measure and $s_{\text {ref }}$ denotes the reference point. Figure 1 shows the hypervolume of a non-dominated set as a gray region.

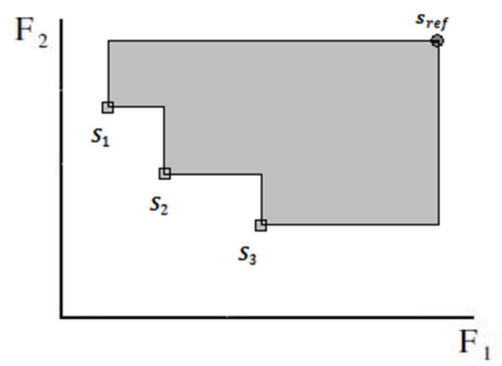

Figure 2. Hypervolume of non-dominated solutions

Fleischer [14] prove that the hypervolume indicator attains its maximum if and only if its nondominated vectors in the objective space equals to its true Pareto-front. Also a set of bounded size which has reached to its maximal hypervolume value should be well-distributed on its true Pareto-front [13]. Respect to a lot of advantages, this measure has one downside which is the computational complexity. It is shown that the exact calculation of the hypervolume is classified in NP-Hard problems [15]. But its drawback could be solved by means of approximation methods and estimative approaches [16].

\subsection{Basic MOEAs}

In the following we will briefly explain some well-known researches in contest of evolutionary multi objective algorithms. As the most popular method, "Nondominated Sorting Genetic Algorithm" NSGA-II proposed by Deb et al. [3], using an elite survival selection mechanism with fast non-dominated sorting algorithm for classification of candidate solutions into domination ranks. NSGA-II adopts crowding distance mechanism for diversity preservation in the Paretofront. In this approach, solutions which belong to the lower (i.e. better) rank and be farther from its neighbors has higher probability to become as a parent and will survive (i.e. assigned higher fitness value). Figure 3 shows the environmental selection in NSGA-II.

As a Second basic method which will describe in this paragraph, so called "S-metric selection evolutionary multi objective algorithm" (SMS-EMOA) proposed by Beume et al [4].The SMSEMOA integrates the fast nondominated sorting approach from NSGA-II with the principle of hypervolume proposed by Zitzler et al. [11] together to reach a higher quality set indicator towards the generation. It is a steady-state algorithm; in each generation one offspring has been generated then combined with parents. In environmental selection, at first, all generated solutions are ranked in some level then eliminate one solution, which contributes the least hypervolume (i.e. indicator) to the worst-ranked. Equation (6) and figure 4 illustrate this matter. 
International Journal of Artificial Intelligence \& Applications (IJAIA), Vol.3, No.1, January 2012

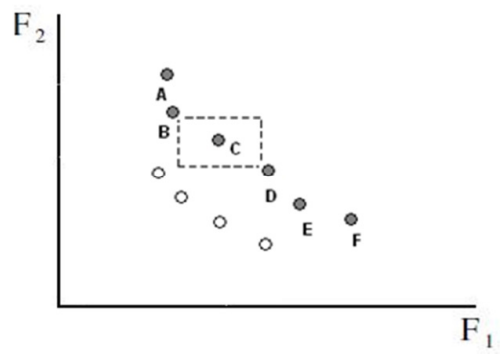

Figure 3. Graphical illustration of crowding distance in NSGA-II [3]

$\operatorname{Min}\left(\mathcal{S}\left(R_{w}\right)-\mathcal{S}\left(R_{w}\{s\}\right)\right), R_{w}=\{s \mid s \in$ higher level $\}$

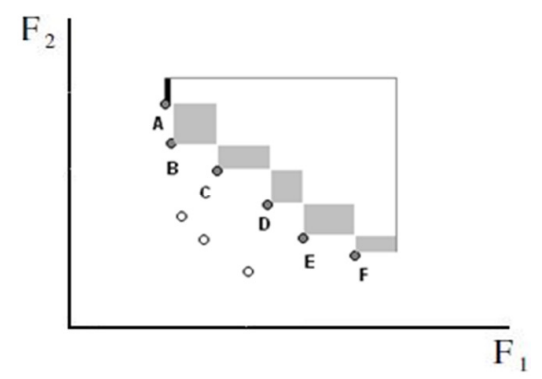

Figure 4. Graphical representation of marginal hypervolume of final non-dominated ranked solutions in environmental selection of SMS-EMOA, for example in this figure the solution A is the best candidate for elimination.

\section{RELATED WORK}

Maintaining population diversity in the solution space recently is one of the important research topics in terms of multi-objective evolutionary algorithms not only because of its advantages but also because there are many open questions in its realm. Several researches have denoted and illustrated some characteristic of new test problems related to diversity of the Pareto-set and compared diversity indicator of non-dominated solutions of existing MOEAs [9, 17].In GDEA proposed by Toffolo and Benini [18] invoked two selection mechanism, nondominated sorting approach from Goldberg [19] as the primary one for ranking and a distance-based measure for preserving diversity of obtained non-dominated set in the solution space. Another approach proposed by Deb and Tiwari [7] in which integrated the crowding distance in the solution space into the nondominated sorting scheme and extended the framework of NSGA-II consequently, make better diversity criterion in the solution space. Shir et al. [8] also proposed in which integrated two set criteria into CMA-ES niching framework to promote solution space diversity. MMEA [9] another new approach, has used a probabilistic model-based multi-objective evolutionary algorithm. In this method, at first, the population is clustered into a number of subpopulations, the principal component analysis (i.e. PCA) approach is used to estimate the dimensionality of the Pareto-set diversity in each subpopulation, and then a probabilistic model of non-dominated solution is built. After this, new offspring is generated based on this model. Diversity preserving in this method is regarded to genetic operators and take advantage of estimation of distribution algorithm (EDA) mechanism.

In the following we explain and characterize one of the newest multi-objective evolutionary algorithm so called, the DIVA (diversity integrating hypervolume-based search algorithm) 
International Journal of Artificial Intelligence \& Applications (IJAIA), Vol.3, No.1, January 2012

proposed by Zitzler et al. [10] in more detail. In DIVA first, diversity of non-dominated solution is calculated respect to the solution space. Then hypervolume of outstanding solutions is computed in the objective space and partitioned in appropriate segments. Each segment of the divided hypervolume is weighted and modified based on diversity indicator values of relevant solutions. Figure 5 shows the mechanism of integration in DIVA. The Rest of this method is similar to other indicator-based evolutionary multi-objective algorithms. By integrating the diversity indicator of non-dominated solutions corresponding to the solution space into hypervolume measure, the search algorithm is encouraged to find more diverse Pareto-set.
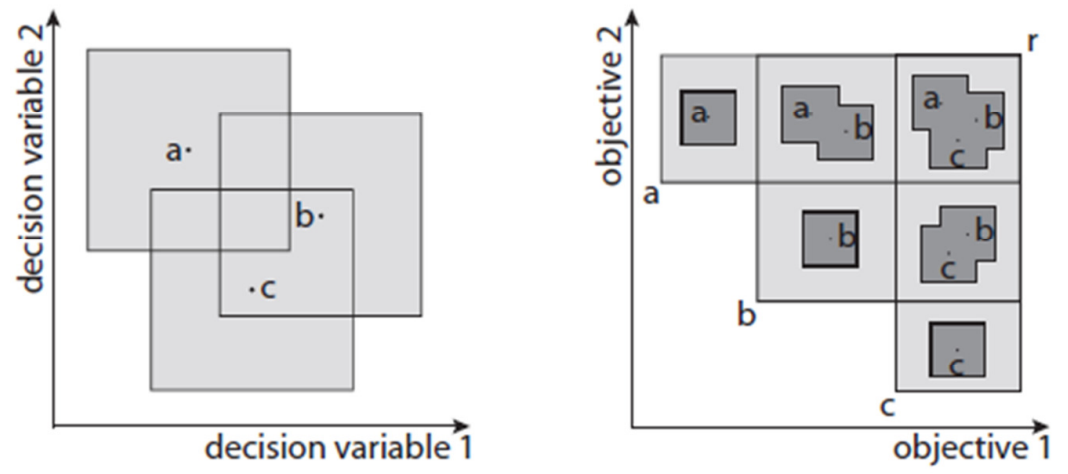

Figure 5. The left figure shows the three solutions in the solution (decision) space with their diversity measure. The right figure shows the hypervolume partitions, where in each partition the relevant diversity is shown qualitatively. This figure is taken from DIVA [10]

According to DIVA, it seems that some contributions make to be better quality of diversity indicator and in addition, modifying the selection mechanism can help to the algorithm for saving time-consumption. In this study a novel diversity indicator is introduced which released from the shape of coverage area and have not any extra parameters relative to DIVA.

\section{THE PROPOSED METHOD}

Before discussing on our method, first we introduce the proposed diversity measure used in this paper and its properties are determined. In addition, all of conditions of any diversity set measures suggested in [20] are elucidated. Then we exhibit how such a diversity measure can be integrated into the hypervolume measure.

Diversity is inherently a property of sets of solutions rather than single solutions; therefore diversity of single solution is not meaningful. In diversity set measurement procedure, the real value is allocated to a relevant subset. In evolutionary algorithms (EAs) diversity measure of a set is based on the pair-wise distance between each elements belong to set. But maximizing any pairwise distance based diversity may not distribute all of elements well on their feasible regain. For example, we show two different one dimensional sets in figure 6, According to this figure, it's clear that the above shape has a higher diversity value according to pair-wise distance then the bottom one, meanwhile this result is not truth, because the elements of set of bottom shape is well-distributed and more diverse form others. 
International Journal of Artificial Intelligence \& Applications (IJAIA), Vol.3, No.1, January 2012

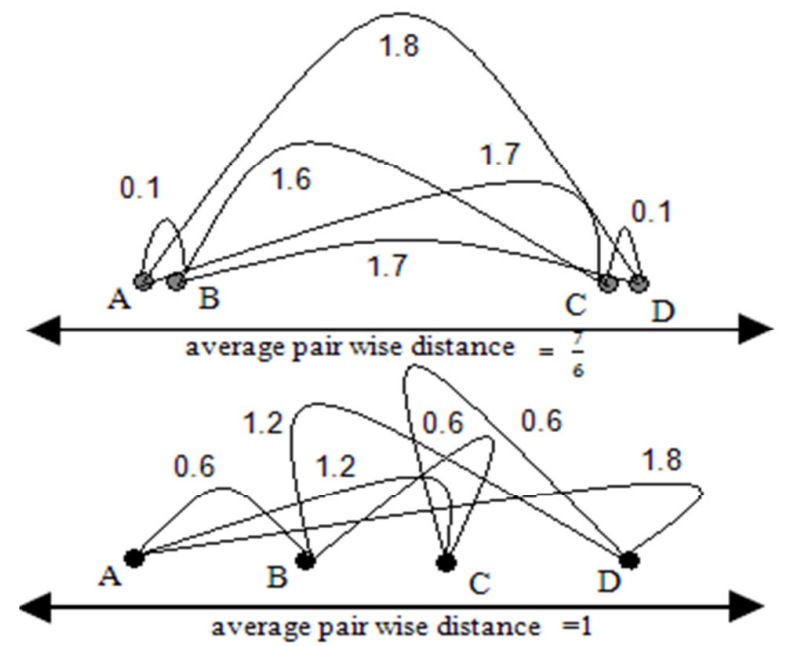

Figure 6. Comparison of traditional diversity indicator (i.e. average pair-wise distance) between two different sets. Although elements of the bottom shape are more divers then the above shape, but assigned lower diversity indicator (e.g. $\frac{(0.6 * 3+1.2 * 2+1.8)}{6}=1$ )

In this paper we introduce the novel diversity mechanism as a first time for estimate dimensionality of non-dominated solutions in the solution space. Moreover this diversity masseur aims at solving the major drawback of pier-wise distance based diversity indicator. Our proposed diversity measure is based on pair-wise distance with some modifications. To calculate the diversity indicator of one set, at first place we compute the geometrical mean value of the set, then elements which far from the mean value are found. Now a circle is drawn around the mean value with specific radii (i.e. radii is computed based on Euclidean distance between the center and the extreme element). As soon as compute the radii, all of elements that hold on the circle or too close to the circle must be eliminate from the set after then the mean value of the modified set is updated and all parameters of generated circle are recalculated. This procedure continued until no element exists in the set. After termination, we sum all radiuses up and proposed this value as a diversity indicator of the set. Figure 7 illustrates the computing of the new diversity measure. In fact, all of which process is performed in solution (feature) space due to indicate how obtained Pareto-set is separable and diverse. The new diversity indicator is evaluated based on sum of sum of these radii and number of generated circle.

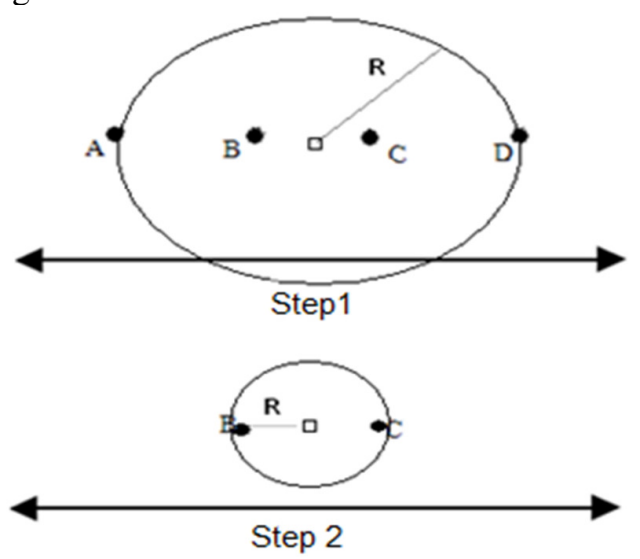

Figure 7. Illustration of the proposed diversity indicator: In each step, members that near to the circle must be eliminated and calculate the mean value of set is calculated again 
That parameter which determines how many of elements are close to circle is crucial and need to be optimized. This parameter plays a role of niching towards procedure of this diversity measure and do not allow number of elements are crowded in specific region, it means that this diversity indicator resolves downside of pair-wise distance indicator, furthermore using sequences of radii makes the set become enlarged. Finally the parameter which indicates number of center is utilized for separability of the set. With integrated all of generated parameters into a unit formula we can see that all of primary conditions of any diversity indicator discussed in [20] will be satisfied.

For getting better result we used a monotonic logarithmic function to adjust obtained value of diversity indicator. Equation (6) shows this function.

proposed Diversity Indicator $=\frac{\log (100) *(1+C+\sqrt{\mathrm{R}})}{\log (100)}$

Where the parameter $\mathrm{C}$ is a number of generated circles and the parameter $\mathrm{R}$ is a sum of obtained radiuses. After definition of the proposed diversity indicator, it is a good time to use this measure and integrated it into multi-objective optimization framework. In this study we take advantage of weighted hypervolume-based MOEAs, due to their suitable properties, also it becomes more popular for solving MOPs [16],[21], [22] recently, furthermore this measure enable us to combine other set preference indicator easily [12]-[20]. As a discussed before, SMS-EMOA [4] is the famous MOEA that use a hypervolume as fitness assignment to each element belong to the latest Pareto-front. Our proposed method weights all parts of marginal hypervolume in SMS-MOEA by each diversity indicator of relevant solution. Hypervolume of each part are weighted based on contribution of relevant non-dominated solution respect to loss diversity indicator from the Pareto-set. By doing this, both objective space set preference (i.e. hypervolume indicator) and solution space set preference (i.e. Pareto-set diversity indicator) can be optimized. In our approach, generated solutions randomly at first, then evaluate and sort all candidate solution based on non-dominated sorting algorithm [3], finally in survival selection scheme, form the latest rank, select one of solution which has a minimum weighted hypervolume indicator value. Block diagram of proposed method is given in figure 8. As a result of reappraisal of the diversity indicator in each elimination procedure at environmental selection, the computational complexity of proposed method is more than basic methods which described in section 2, but this downside can be ignored due to most of evolutionary multi-objective algorithms are run in offline mode. 


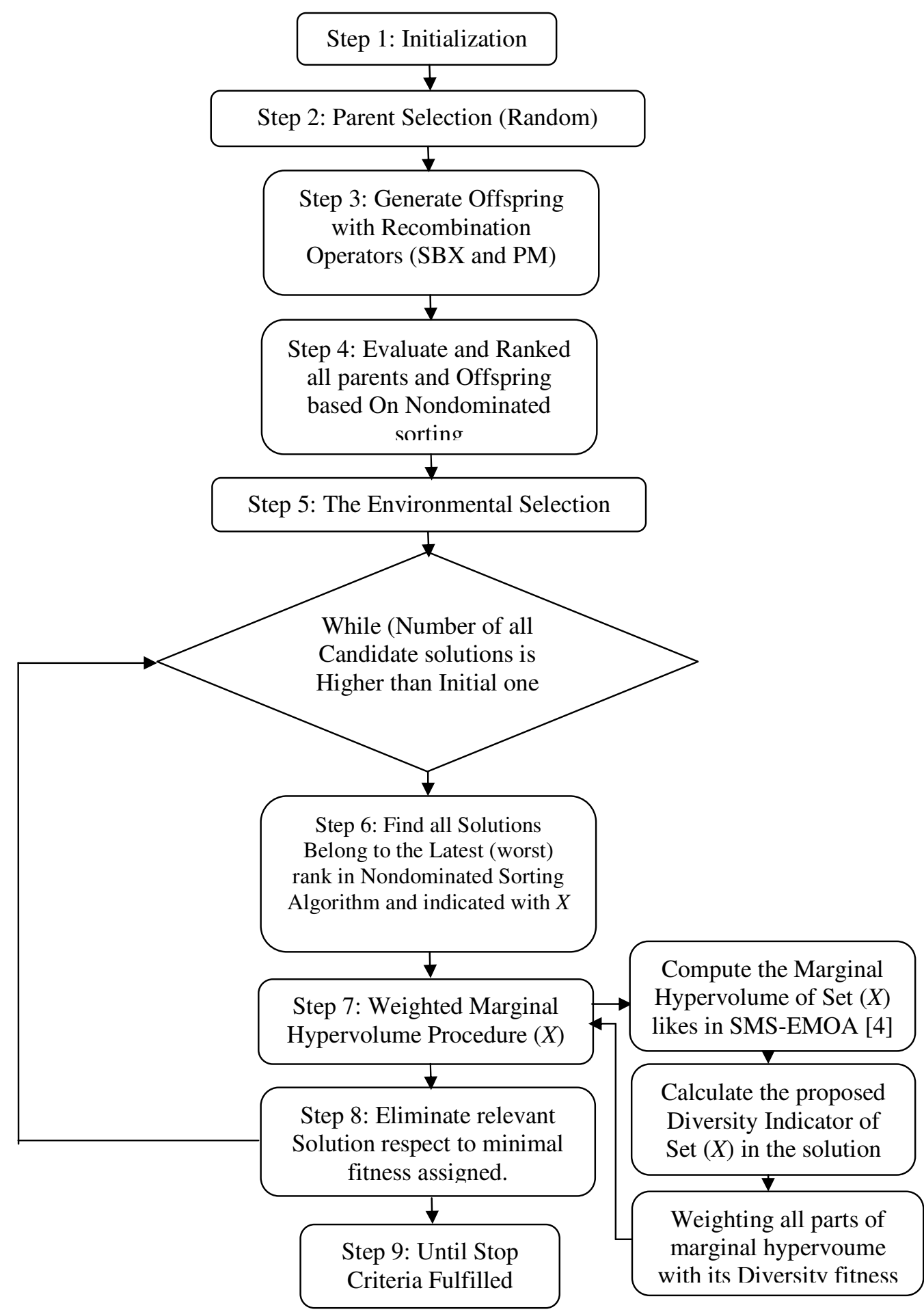

Figure 8. Block Diagram of proposed method 
International Journal of Artificial Intelligence \& Applications (IJAIA), Vol.3, No.1, January 2012

Another novelty of this paper is fitness assignment strategy in the environmental selection. As soon as marginal hypervolume is computed (i.e. hypervolume contribution regarding loss of relevant solution in the objective space), the diversity measure of each solution is calculated (i.e. regarding the losing relevant solution from Pareto-set in the solution space) and then two indicators are embedded. So worst members form below average population are removed in environmental selection. Figure 9 shows the embedding of diversity indicator to the hypervolume criterion.
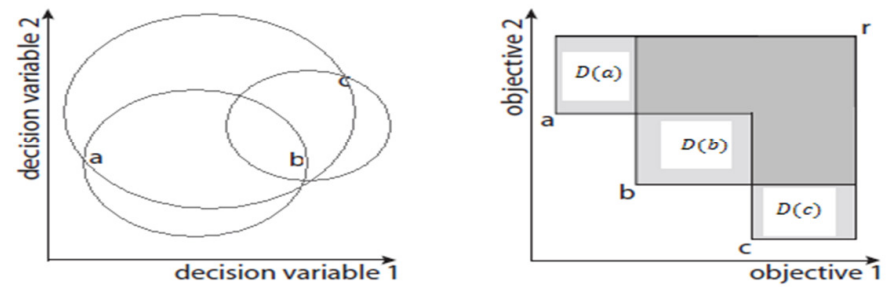

$$
\left\{\begin{array}{l}
D(a)=D\{a, b, c\} \backslash D\{b, c\} \\
D(b)=D\{a, b, c\} \backslash D\{a, c\} \\
D(c)=D\{a, b, c\} \backslash D\{a, b\}
\end{array}\right.
$$

Figure 9: fitness assignment in proposed method. In this case, D(.)is denoted as a diversity indicator

\section{THE EXPERIMENTAL RESULT}

This section is devoted to the evaluation of proposed method (WMH-MOEA) in both objective and solution space. For that, we have chosen several test problems taken from the specialized literature in order to assess how competitive WMH-MOEA is, we decided to compare it against two algorithms that are representative of the state-of-the- art, namely SMS-EMOA and NSGA-II.

\subsection{Test Problems}

All of test problems which have been adopted in this paper are continues bi-objective minimization problems [23-26]. None of them have any inequality or equality constraints. Also they belong to type 1 of test problems corresponding to the classification of test suite in [9].Table 1 shows all used test sites and their properties.

Table 1. Test problems used in this paper, $\mathrm{n}$ is the number of decision variables of the solution

\begin{tabular}{|c|c|c|c|}
\hline Problem & Obiective functions & bound & \\
\hline Omni-Test & $f_{1}(x)=\sum_{i=1}^{n} \sin \Pi x_{i}, \quad f_{2}(x)=\sum_{i=1}^{n} \cos \Pi x_{i}$ & $0<x<6$ & 6 \\
\hline & $f_{1}(x)=\left(\sum_{i=1}^{n}\left|x_{i}\right|\right)^{r} \cdot n^{-\gamma}, f_{2}(x)=\left(\sum_{i=1}^{n}\left|x_{i}\right|-1\right)^{r} \cdot n^{-\gamma}$ & $r=1$ & \\
\hline EBN-1est & & $-2 \leq x_{i} \leq 2$ & 0 \\
\hline Two-On-One & $\begin{array}{l}f_{1}(x)=x_{1}^{4}+x_{2}^{4}-x_{1}^{2}+x_{1}^{2}+x_{2}^{2}-C x_{1}^{1} x_{2}+\mathrm{d} x_{1}+20 \\
f_{2}(x)=\left(x_{1}-k\right)^{2}+\left(x_{2}-l\right)^{2}\end{array}$ & $\begin{array}{c}-4 \leq x_{i} \leq 4 \\
k=0, l=0 \\
\mathrm{c}=10, \mathrm{~d}=0.25\end{array}$ & 2 \\
\hline Lame Super Spheres & $\begin{array}{c}f_{1}(x)=(1+r) \cdot\left(\cos x_{1}\right), f_{2}(x)=(1+r) \cdot\left(\sin x_{1}\right) \\
r=(\sin \Pi d)^{2}, d=\frac{1}{n-1} \sum_{i=2}^{n} x_{i}\end{array}$ & $\begin{aligned} 0 & \leq x_{1} \leq \frac{\Pi}{2} \\
1 & \leq x_{i} \leq 5 \\
\text { For } i & =2 \text { to }\end{aligned}$ & 6 \\
\hline ZDT6 & $\begin{array}{c}f_{1}(x)=1-e^{-4 x_{1}} \cdot \sin ^{6}\left(6 \Pi x_{1}\right), f_{2}(x)=g(x) \cdot\left(1-\left(\frac{f_{1}}{g(x)}\right)^{2}\right) \\
g(x)=1+9\left(\frac{\sum_{i=2}^{n} x_{i}}{n-1}\right)^{0.25}\end{array}$ & $0 \leq x_{i} \leq 1$ & 6 \\
\hline
\end{tabular}




\subsection{Parameter Settings}

In the following we explain parameter settings of methods that must be compared. Proposed method was run on test suite whit population size as well as the number of offspring was set to 30 and reference vector is 10 for computing the S-metric. Variation operators are SBX [27] and polynomial mutation [28] which are similar to NSGA-II [3]. Our algorithm was run for 500 generations. The NSGA-II algorithm was proposed by Deb et al. It is characterized by a Pareto ranking of the individuals and the use of a crowding distance as density estimator. Specifically, we used the real-coded version of the algorithm and the parameter settings proposed in [3]. The operators for recombination and mutation are SBX and polynomial mutation, with distribution indexes of $\eta c=20$ and $\eta \mathrm{m}=20$, respectively. A recombination probability of $\mathrm{P}_{c}=0.9$ and a mutation probability $\mathrm{p}_{\mathrm{m}}=0.1$ are used. The population size is 30 individuals. The algorithm stops after 500 generation. Another method that used for comparing with proposed method is SMS-EMOA. We adjust the model of steady state in this method to $(\mu+\mu)$-EA for decency between algorithms and in the following have addressed as the modified version of SMS-EMOA to Original IBEA.

\subsection{Performance Metrics}

To exploit the advantage of proposed method we used the S-metric as a measure to convergence of Pareto-front. In order to assess the enhancing of diversity in the decision space we calculated the average distances between solutions belongs to Pareto-set. Hypervolume or S-metric is a compliant measure related to Pareto dominance and computes the volume of the dominated region, demarcated by a given set of points (i.e. non-dominated solutions in objective space), and a reference vector. Higher value of this measure indicates that better performance corresponds to the objective space criteria and it means that the set of non-dominated solutions approximates close to the true Pareto-front.

Also we look at a commonly used diversity indicator to verify our findings in terms of population diversity in the solution space, so called pair-wise distance. Pair-wise distance computes the mean of all distances between members of Pareto-set in the solution space. Mathematically definition of pair-wise distance is:

$$
\text { Pair-wise Distance }=\frac{\sum_{i=1}^{n} \sum_{j=\mathrm{i}}^{\mathrm{n}} \mathrm{x}_{\mathrm{ij}}}{n(n-1) / 2}
$$

where $x_{i j}$ is the distance between $i$-th and $j$-th solution in the solution space. It is clear that if $\mathrm{i}=\mathrm{j}$ then $\mathrm{x}_{\mathrm{ij}}$ must be equal to zero. Furthermore we use the proposed diversity indicator described in past section (Equation. 6) to better demonstrate of capability of MOEAs corresponds to diversity of Pareto-set in the solution space.

The New Diversity Indicator $=$ proposed Diversity Indicator $($ Pareto-Set $)$

\subsection{The Experimental Result}

All numerical results of this paper are average of ten independent runs and implemented by MATLAB software. The experiment was reported for proposed method and all of its competitors as shown in Table 2. The S-metric as a performance measure in terms of objective space and pair- 
International Journal of Artificial Intelligence \& Applications (IJAIA), Vol.3, No.1, January 2012

wise distance as diversity indicator of Pareto-set are two popular preference measures in this table. According to Table 2, it is obvious that in all cases, the proposed method is better (i.e. higher value) than its competitors correspond to diversity indicator of population in the solution space. For better comprehensive analyzes on results, the new proposed diversity indicator is used to assess which algorithms on test problems have higher diversity measure and shown in Table 3. Results in this table are consistent with outcomes from Table 2 and they are showing that, the WMH-MOEA has a better (higher value) diversity indicator then Original IBEA and NSGA-II according to the solution space. Pareto-set of all test problems generated by WMH-MOEA and Original IBEA are plotted in Table 4 respectively as a graphically simulating of outcomes in the solution space. In this table Horizontal axis includes the number of decision variable of test problems and vertical axis includes the values of each Pareto optimal solution in its feasible range. This table exactly exhibits high performance of proposed method, also explicitly demonstrates all numerical results of Table 2 and Table 3. For example, Omni test function as a case study; due to its attributes of Pareto-optimal set (i.e. Pareto-set could be partitioned into subsets where the images of each subset are identical), it's obvious from corresponding figures that, non-dominated solutions induced by WMH-MOEA are more separate from other, and generated Pareto-optimal solutions has a higher diversity indicator due to ability of crowding more area of its feasible region in solution space. Therefore this method can find more subset of Pareto-optimal set in contrast to Original MOEA. As another case, TWO-ON-ONE test problem in which Pareto front is multiply covered by disjoint parts of the Pareto set. With comparing figures about this problem, we can see that, our proposed method outperforms the Original IBEA in terms of solution space diversity criterion. Finally in ZDT6 case study, it is clear that, generated set of non-dominated solutions by means of proposed method is more diversified then competitive method as a result of obtaining more distinct non-dominated solutions in Pareto-set.

Pareto-front of each test problems was plotted to comprehensive better comparison between MOEAs in figure 10-14 respectively. Our method is better approximate the true Pareto-front in some cases, but due to improving solution space diversity measure; diversity of Pareto-front has been defected. In these figures, the star points indicate the Pareto-front by means of proposed method and the circle points indicate the Pareto-front of test problems by Original IBEA. At the end, with comparison of simulated results of Pareto-set and Pareto-front together; we can see that the proposed method can reach to the higher quality measure in Pareto-set diversity.

Table 2. Results of S-metric and Pair-wise Distance of the proposed method and its competitors on test problems; better results was bolded

\begin{tabular}{|c|c|c|c|}
\hline \multirow{2}{*}{ MOPs } & \multicolumn{3}{|c|}{ Algorithms } \\
\cline { 2 - 4 } & $\begin{array}{c}\text { The Proposed Method: } \\
\text { WMH-MOEA }\end{array}$ & Original IBEA & NSGA-II \\
\hline \multirow{3}{*}{ Omni-Test } & S-metric:247.15 & S-metric:247.60 & S-metric:246.99 \\
& Pair-wise Distance: & Pair-wise Distance: \\
& S-metric:97.80 & S-metric:97.69 & S-metric:98.76 \\
\hline \multirow{2}{*}{ EBN } & Pair-wise Distance: 1.12 & Pair-wise Distance: & Pair-wise Distance: \\
& S-metric:20.44 & S-metric:20.55 & S-metric:20.25 \\
\hline Two-On- & Pair-wise Distance: 1.01 & Pair-wise Distance: & Pair-wise Distance: \\
One & S-metric:99.19 & S-metric:99.20 & S-metric:99.13 \\
\hline Lame & Pair- & Pair-wise Distance: & Pair-wise Distance: \\
Super & Pair-wise Distance: 3.12 & $\mathbf{2 . 0 6}$ & 2.28 \\
\hline
\end{tabular}


International Journal of Artificial Intelligence \& Applications (IJAIA), Vol.3, No.1, January 2012

\begin{tabular}{|c|c|c|c|}
\hline \multirow{2}{*}{ MOPs } & \multicolumn{3}{|c|}{ Algorithms } \\
\cline { 2 - 4 } & $\begin{array}{c}\text { The Proposed Method: } \\
\text { WMH-MOEA }\end{array}$ & Original IBEA & NSGA-II \\
\hline \multirow{3}{*}{ ZDT6 } & S-metric:96.77 & S-metric:96.78 & S-metric:96.74 \\
& Pair-wise Distance: $\mathbf{0 . 3 3}$ & Pair-wise Distance: & Pair-wise Distance: \\
& & 0.08 & 0.13 \\
\hline
\end{tabular}

Table 3.Comparison between results of MOEAS for the proposed diversity indicator on test problems; higher value means better performance respect to solution space diversity. Better result was bolded.

\begin{tabular}{|c|c|c|c|}
\hline \multirow{2}{*}{ MOPs } & \multicolumn{3}{|c|}{ The New Diversity Indicator } \\
\cline { 2 - 4 } & $\begin{array}{c}\text { The Proposed } \\
\text { Method: } \\
\text { WMH-MOEA }\end{array}$ & Original IBEA & NSGA-II \\
\hline Omni-Test & $\mathbf{1 . 6 6 2 4}$ & 1.5914 & 1.5021 \\
\hline EBN & $\mathbf{1 . 6 4 0 2}$ & 1.5763 & 1.6002 \\
\hline Two-On-One & $\mathbf{1 . 5 5 4 4}$ & 1.4977 & 1.4980 \\
\hline Lame Super spheres & $\mathbf{1 . 6 7 3 9}$ & 1.5601 & 1.5291 \\
\hline ZDT6 & $\mathbf{1 . 5 4 2 3}$ & 1.2348 & 1.2566 \\
\hline
\end{tabular}

Table 3. parallel coordinate plot of 30 individual of population in the Pareto-set of test problems in the solution (decision) space; horizontal axis indicates number of decision variable in the solution space, vertical axis indicate feasible value of each decision variable in the solution space.

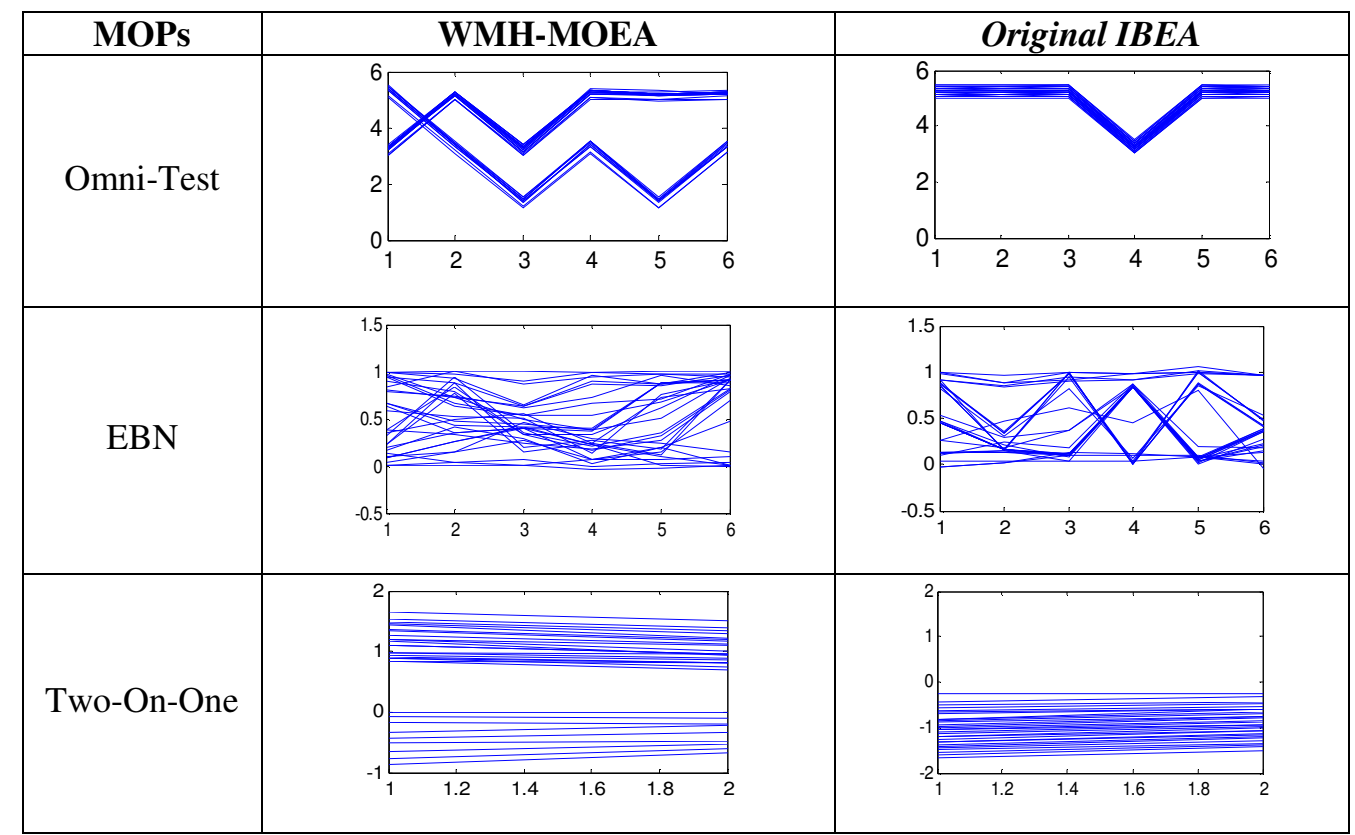


International Journal of Artificial Intelligence \& Applications (IJAIA), Vol.3, No.1, January 2012

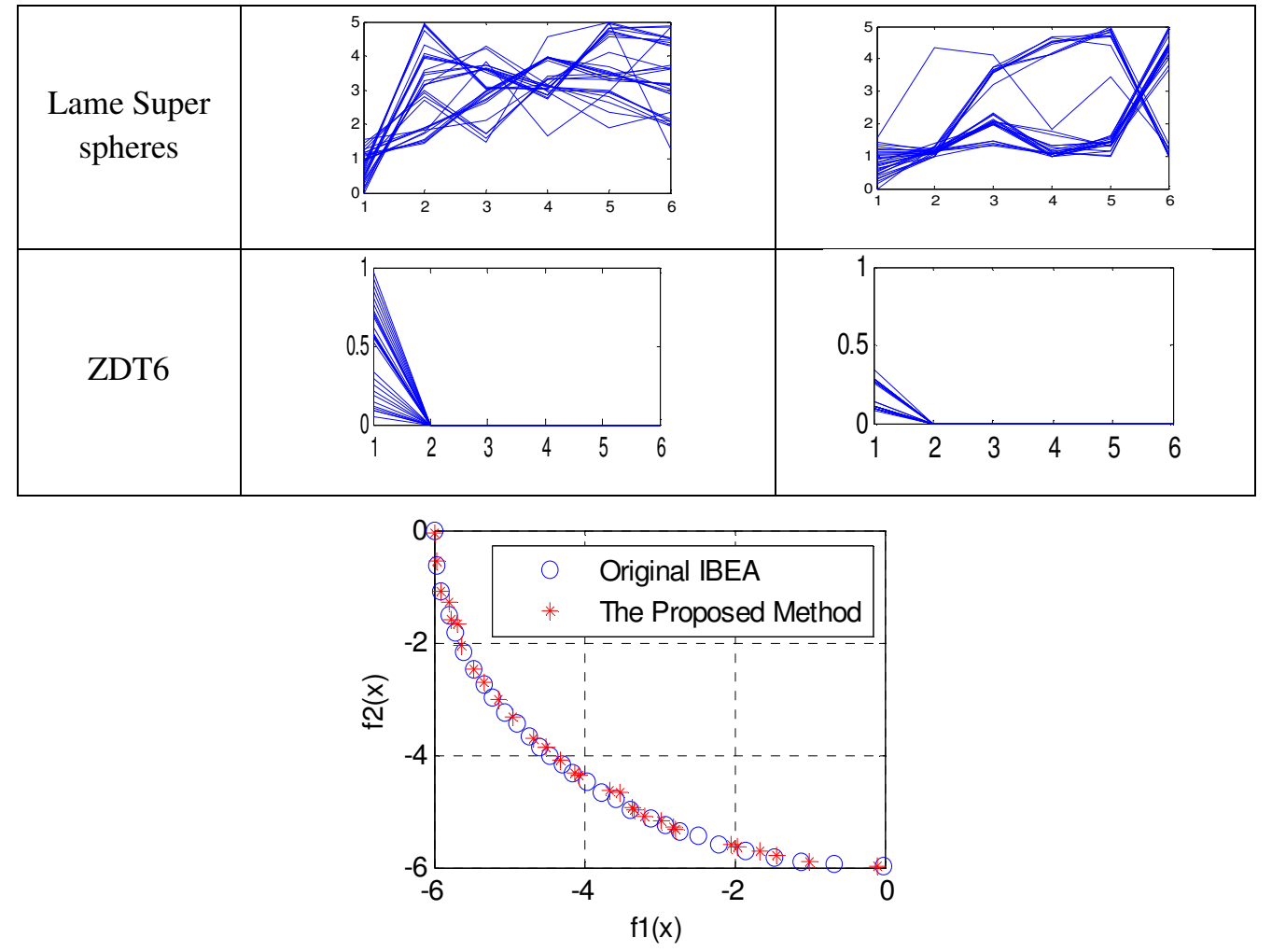

Figure 10. Pareto-front of Omni Test problem.

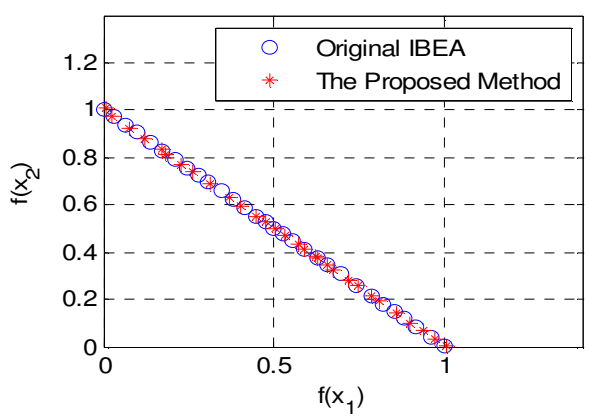

Figure 11. Pareto-front of EBN Test problem

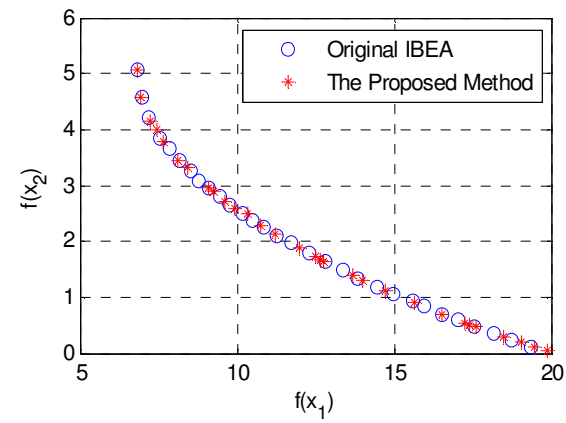

Figure 12. Pareto-front of TWO-ON-ONE Test problem 
International Journal of Artificial Intelligence \& Applications (IJAIA), Vol.3, No.1, January 2012

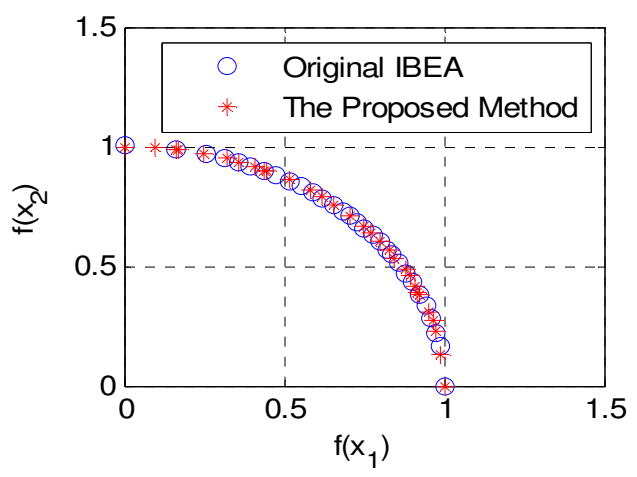

Figure 13. Pareto-front of Lame Super spheres Test problem

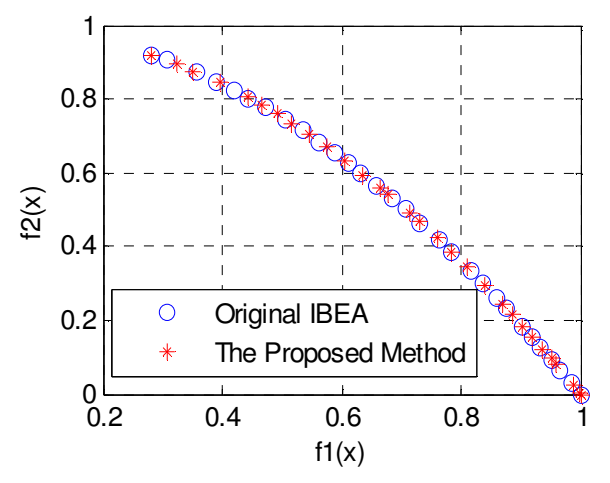

Figure 14. Pareto-front of ZDT6 Test problem

\section{CONCLUSION AND FUTURE WORK}

Diversity preservation is an important notion and has played a major role in terms of multi objective optimization. But most of researches just have focused on diversity in the objective space. In this paper at first, we introduced the novel diversity criterion, then to achieve to the main objective of this paper and obtaining the Pareto-set with higher diversified populations, we integrated the proposed diversity indicator as a weighted method into hypervolume based multiobjective search. By utilizing the diversity mechanism in the environmental selection, solution which preserving higher fitness in both objective and solution spaces becomes survived to the next generation. The results on test problems generated by proposed method are significant better than other MOEAs correspond to solution space diversity. According to figures that contain diagrammatic Pareto-set, it is obvious that, our proposed method be able to find diversified set of solutions in the decision space.

As a future work we suggest to study on solution space diversity of many objective optimization problems and apply this framework on these problems. Also using of estimation algorithms and other intelligent mechanism to find and discover more area of Pareto-optimal set of solutions in less time consumption is recommended. Our future research has devoted to the evolvement of adaptive strategies and takes advantage of machine learning techniques to optimize parameters of the proposed method, consequently more accurate result will be acquired even if only few fitness function evaluations can be take place. 
International Journal of Artificial Intelligence \& Applications (IJAIA), Vol.3, No.1, January 2012

\section{ACKNOWLEDGEMENTS}

The first author would like to thank Miss. Z.Khorshidpour, Ph.D. student of artificial intelligent at Department of CSE and IT in Shiraz University, for useful suggestion.

\section{REFERENCES}

[1] C. A. C. Coello, D. A. V. Veldhuizen and G. B. Lamont, Evolutionary Algorithms for Solving MultiObjective Problems. 2rd ed., Springer- Verlag, 2007.

[2] K. Deb, Multi-Objective Optimization Using Evolutionary Algorithms. New York: Wiley, 2001.

[3] K. Deb, A. Pratap, S. Agarwal and T. Meyarivan, "A Fast and Elitist Multiobjective Genetic Algorithm: NSGA-II,” IEEE Transactions on Evolutionary Computation, vol. 6, no. 2, $\quad$ pp. 182197 (2002).

[4] N. Beume, B. Naujoks, and M. Emmerich, "SMS-EMOA: Multiobjective Selection Based on Dominated Hypervolume," European Journal on Operational Research, 181, pp. 1653-1659 2007.

[5] K. Deb and N. Srinivas, "Multiobjective Optimization Using Nondominated Sorting in Genetic Algorithms,', Evolutionary Computation, vol. 2, no. 3, pp.221-248,1994

[6] A. Eiben and J. Smith, Introduction to Evolutionary Computing. Springer, Berlin, 2003. ISBN 3-54040184-9.

[7] K. Deb and S. Tiwari," Omni-optimizer: A Procedure for Single and Multi-objective Optimization," In: Evolutionary Multi-Criterion Optimization (EMO, 05), 2005, Springer Berlin, pp. 47-61.

[8] O. M. Shir, M. Preuss, B. Naujoks, and M. Emmerich, "Enhancing decision space diversity in evolutionary multiobjective algorithms," In Conference on Evolutionary Multi-Criterion Optimization (EMO, 09), 2009, pp. 95-109.

[9] A. Zhou, Q. Zhang, and Y. Jin, "Approximating the set of pareto optimal solutions in both the decision and objective spaces by an estimation of distribution algorithm," IEEE Transactions on Evolutionary Computation, vol. 13, no. 5, pp. 1167-1189, 2009.

[10] T. Ulrich, J. Bader, and E. Zitzler, "Integrating Decision Space Diversity into Hypervolume-based Multiobjective Search," In Genetic and Evolutionary Computation Conference (GECCO 2010), New York, NY, USA, 2010, pp. 455-462.

[11]E. Zitzler, L. Thiele, M. Laumanns, C. M. Fonseca, and V. Grunert da Fonseca, 'Performance assessment of multiobjective optimizers: an analysis and review,' IEEE Transactions on Evolutionary Computation, vol. 7, no. 2, pp.117-132, 2003.

[12] E. Zitzler, D. Brockho_, and L. Thiele, "the hypervolume indicator revisited: On the design of Pareto-compliant indicators via weighted integration,' In Proc. Fourth International Conference on Evolutionary Multi-Criterion Optimization (EMO '07), 2007, Vol. 4403 of LNCS, pp. 862.

[13] N. Beume, B. Naujoks, and M. Emmerich, "An EMO algorithm using the hypervolume measure as selection criterion,” In Proc. Conf. Evol Multi-Criterion Optimization (EMO '05), 2005, LNCS vol. 3410. New York: Springer-Verlag, pp. 62-76.

[14] M. Fleischer, The Measure of Pareto Optima, "Applications to Multi-objective Metaheuristics," Evolutionary Multi-Criterion Optimization (EMO, 03), 2003, LNCS, vol. 2632, Springer, Berlin, 2003, pp. 519-533. Springer, 2008.

[15] K. Bringmann and T. Friedrich, 'Approximating the volume of unions and intersections of highdimensional geometric objects," In International Symposium on Algorithms and Computation (ISAAC), volume 5369 of Lecture Notes in Computer Science, pages 436-447.

[16]J. Bader and E. Zitzler, "HypE: An Algorithm for Fast Hypervolume-Based Many-Objective Optimization," Evolutionary Computation, vol. 19, no. 1, pp.45-76, 2011.

[17] G Rudolph, B Naujoks and, M Preuss, 'Capabilities of EMOA to Detect and Preserve Equivalent Pareto Subsets,' In: Evolutionary Multi-Criterion Optimization (EMO, 07), 2007, Springer, Berlin, pp. 36-50.

[18] A. Toffolo and E. Benini, "Genetic diversity as an objective in multi-objective evolutionary algorithms,' Evolutionary Computation, vol. 11, no. 2, pp. 151-167, 2003.

[19] D. E. Goldberg, Genetic Algorithms in Search, Optimization and Machine Learning. Addison-Wesley Publishing Company, Reading, Massachusetts, 1989. 
International Journal of Artificial Intelligence \& Applications (IJAIA), Vol.3, No.1, January 2012

[20] T. Ulrich, J. Bader and L. Thiele,’Defining and Optimizing Indicator-based Diversity Measures in Multiobjective Search," parallel problem solving from nature (PPSN), 2010, pp. 707-717.

[21]E. Zitzler and S. K"unzli, "'Indicator-based Selection in Multiobjective Search,' Parallel Problem Solving from Nature - PPSN VIII pages 832-842, Birmingham, UK, September 2004. SpringerVerlag. Lecture pages 832-842, Birmingham, UK, September 2004. Springer-Verlag. Lecture.

[22]E. Zitzler, L. Thiele, and J. Bader,’On Set-Based Multiobjective Optimization,” IEEE Transactions on Evolutionary Computation, vol. 14, no. 1, pp. 58-79, 2010.

[23]E. Zitzler and L. Thiele, "Multiobjective Optimization Using Evolutionary Algorithms-A comparative case study," In Conference on Parallel Problem Solving from Nature (PPSN), 1998, pp. 292-304.

[24] M. Emmerich and A. Deutz, "Test Problems Based on Lame Superspheres," In: Evolutionary MultiCriterion Optimization (EMO, 07), 2007, Springer, Berlin , pp. 922-936.

[25] E. Zitzler, K. Deb and L. Thiele, "Comparison of Multiobjective Evolutionary Algorithms: Empirical Results,” Evolutionary Computation, vol. 8, no. 2, pp. 173-195, Summer 2000.

[26] M. Preuss, B. Naujoks and G. Rudolph, "Pareto Set and EMOA Behavior for Simple Multimodal Multiobjective Functions," In: Parallel Problem Solving from Nature (PPSN), Springer, Berlin (2006), pp. 513-522.

[27] K. Deb and R. B. Agrawal, "Simulated binary crossover for continuous search space," Complex Syst., vol. 9, no. 2, pp. 115-148, 1995.

[28] K. Deb and M. Goyal, "A combined genetic adaptive search (GeneAS) for engineering design," Comput. Sci. Inform., vol. 26, no. 4, pp. 30-45, 1996.

\section{Authors}

KamyabTahernezhadiani received the B.Sc. degree in computer engineering from Azad University of Hamedan. He is currently a M.Sc. student in Artificial intelligence at Shiraz University. His research interests include machine learning, multi-objective evolutionary computing and bio-inspired algorithms.

Dr. Ali Hamzeh received his Ph.D. in artificial intelligence from the Iran University of Science and Technology. He is lecturer in deportment of Computer Science Engineering and Information Technology. $\mathrm{He}$ is co-author of several articles in security and optimization and reviewer in reputed journals.

Dr.Sattar Hashemi received his Ph.D. degree in Computer Engineering from the Iran University of Science and Technology. He is now an assistant professor in CSE and IT department at Shiraz University. His research interests include machine learning, social web mining, and game theory. 